\title{
SMOOTH NORMS THAT DEPEND LOCALLY ON FINITELY MANY COORDINATES
}

\author{
PETR HÁJEK
}

(Communicated by Dale Alspach)

\begin{abstract}
We characterize separable normed spaces that admit equivalent $C^{\infty}$-smooth norms depending locally on finitely many coordinates. It follows, in particular, that such norms exist on any normed space with countable algebraic basis.
\end{abstract}

We use the method of Talagrand operators developed by Haydon in [8] and certain integral convolution techniques [2], [13] to characterize separable normed spaces that admit $C^{\infty}$-smooth norms depending locally on finitely many coordinates as spaces that admit norms with countable boundaries. As corollaries we obtain improvements on some results of Fonf [3] and Vanderwerff [14] and a new simple proof of a result of Haydon on $C^{\infty}$-smooth renormings of spaces of continuous functions on countable compact sets.

Our results should be compared to the result of Godun, Lin, and Troyanski in [5] where it is shown that every separable Banach space can be given an equivalent norm, the unit ball of which contains countably many strongly extreme points. From this result and Theorem 1 below it follows that strongly extreme points need not necessarily form a boundary of $B_{X^{*}}$.

We will use the notation standard in Banach space theory. In particular, $B_{X}$ and $S_{X}$ will denote respectively the unit ball and the unit sphere of a Banach space $X$. We say that $\|\cdot\|$ depends locally on finitely many coordinates if for each $x \in S_{X}$ there exist an open neighbourhood $O$ of $x$, a finite set $\left\{x_{1}^{*}, \ldots, x_{k}^{*}\right\} \subset X^{*}$, and a function $f: \mathbb{R}^{k} \rightarrow \mathbb{R}$ such that

$$
\|y\|=f\left(x_{1}^{*}(y), \ldots, x_{k}^{*}(y)\right) \text { for } y \in O .
$$

If $(X,\|\cdot\|)$ is a normed space, the set $B \subset S_{X^{*}}$ is called a boundary of $(X,\|\cdot\|)$ if for each $x \in S_{X}$ there exists $x^{*} \in B$ such that $x^{*}(x)=1$.

Theorem 1. Let $(X,\|\cdot\|)$ be a normed space. TFAE:

(i) $X$ admits an equivalent norm having a countable boundary.

(ii) $X$ admits an equivalent norm with a boundary $B$, such that there is a sequence $\left\{K_{n}\right\}_{n \in N}$ of norm compact sets in $X^{*}$ satisfying $B \subset \bigcup_{n \in N} K_{n}$.

Received by the editors October 13, 1993 and, in revised form, June 15, 1994.

1991 Mathematics Subject Classification. Primary 46B03, 46B10, 46B20. 
(iii) $X$ is separable and admits an equivalent norm depending locally on finitely many coordinates.

(iv) $X$ is separable and admits an equivalent norm that is $C^{\infty}$-smooth except at the origin and depends locally on finitely many coordinates.

Proof. (iv) $\Rightarrow$ (iii) is trivial.

(iii) $\Rightarrow$ (ii) Let $\|\cdot\|$ be a norm on $X$ that depends locally on finitely many coordinates. Since $S_{X}$ is separable, it is Lindelöf. Therefore there exist a system $\left\{S_{n}\right\}_{n \in N}$ of open sets in $S_{X}$, a system $\left\{\Phi_{n}\right\}_{n \in N}$ of finite subsets of $S_{X^{*}}, \boldsymbol{\Phi}_{n}=\left\{\varphi_{1}^{n}, \ldots, \varphi_{k_{n}}^{n}\right\}$, and a system of functions $\left\{f_{n}\right\}_{n \in N}, f_{n}: \mathbb{R}^{k_{n}} \rightarrow \mathbb{R}$ such that the following hold true:

(a) $S_{X} \subset \bigcup_{n \in N} S_{n}$.

(b) $\|y\|=f_{n}\left(\varphi_{1}^{n}(y), \ldots, \varphi_{k_{n}}^{n}(y)\right)$ for $y \in S_{n}$.

Consider the duality mapping $J: S_{X} \rightarrow \exp \left(S_{X^{*}}\right)$. Denote

$$
K_{n}=\operatorname{span}\left(\varphi_{1}^{n}, \ldots, \varphi_{k_{n}}^{n}\right) \cap S_{X^{*}} \quad \text { for } n \in \mathbb{N} .
$$

$K_{n}$ 's are norm compact sets. For arbitrary $x \in S_{n}, x^{*} \in J(x)$ we have $x^{*}(y) \leq$ $\|y\| \leq 1$ for $y \in S_{n}$. So $x^{*}(y) \leq f\left(\varphi_{1}^{n}(y), \ldots, \varphi_{k_{n}}^{n}(y)\right) \leq 1$ for $y \in S_{n}$. If $h \in \bigcap_{i=1}^{k_{n}} \operatorname{Ker} \varphi_{i}^{n}$ and $\|h\|$ is small enough, then

$$
\|x \pm h\|=f\left(\varphi_{1}^{n}(x \pm h), \ldots, \varphi_{k_{n}}^{n}(x \pm h)\right)=f\left(\varphi_{1}^{n}(x), \ldots, \varphi_{k_{n}}^{n}(x)\right)=1 .
$$

Thus

$$
x^{*}(x \pm h)=1 \pm x^{*}(h) \leq 1
$$

and we have $h \in \operatorname{Ker} x^{*}$. Altogether, $\bigcap_{i=1}^{k_{n}} \operatorname{Ker} \varphi_{i}^{n} \subset \operatorname{Ker} x^{*}$, therefore $x^{*} \in$ $K_{n}$. Hence $J(y) \subset K_{n}$ for $y \in S_{n}$. In combination with (a) we obtain $J\left(S_{X}\right) \subset$ $\bigcup_{n \in N} K_{n}$ and we are done.

(ii) $\Rightarrow$ (i) Let $\|\cdot\|$ be a norm as in (ii). First note that we can replace each $K_{n}$ by $K_{n} \cap B_{X^{*}}$, so we can wlog assume that $K_{n} \subset B_{X^{*}}$ for each $n \in N$. Take a decreasing sequence $\left\{\varepsilon_{n}\right\}_{n \in N}$ such that $\varepsilon_{1}<1, \varepsilon_{n} \downarrow 0$. Then there exist an increasing sequence $\left\{i_{n}\right\}_{n \in N}$ of integers with $i_{1}=1$ and a mapping $I: N \rightarrow B_{X^{*}}$ such that $I\left(\left[i_{n}, i_{n+1}\right]\right)$ forms an $\frac{\varepsilon_{n}}{4}$-net in $K_{n}$. This mapping gives rise to a linear mapping $M: X \rightarrow l_{\infty}(\mathbb{N})$ defined by the formula

$$
M(x)(n)=\left(1+\varepsilon_{k}\right) I(n)(x), \quad \text { where } n \in\left[i_{k}, i_{k+1}\right) .
$$

Take an arbitrary $x \in X,\|x\|=1$. Then $|I(n)(x)| \leq 1$ for each $n \in \mathbb{N}$ and thus $\lim \sup _{n \rightarrow \infty} M(x)(n) \leq 1$. In particular, $\|M(x)\|_{\infty} \leq 1+\varepsilon_{1}$. On the other hand there exist $n_{0} \in \mathbb{N}$ and an element $b \in B$ such that $b \in K_{n_{0}}, b(x)=1$. Hence for some $m \in\left[i_{n_{0}}, i_{n_{0}+1}\right)$ we have

$$
\|I(m)-b\|^{*}<\frac{\varepsilon_{n_{0}}}{4}
$$

and so $I(m)(x) \geq 1-\frac{\varepsilon_{n_{0}}}{4}$. Consequently

$$
M(x)(m) \geq\left(1+\varepsilon_{n_{0}}\right)\left(1-\frac{\varepsilon_{n_{0}}}{4}\right) \geq 1+\frac{\varepsilon_{n_{0}}}{2} .
$$

This proves that $M$ is an isomorphism from $X$ onto some linear subspace of $l_{\infty}(N)$. Since $\limsup _{n \rightarrow \infty} M(x)(n) \leq 1$, we have that $M(x)$ is always an element of $l_{\infty}(N)$ attaining its norm on $N$. If we define

$$
\|x\|_{1}=\|M(x)\|_{\infty}
$$


then the set $\left\{M^{*}\left(e_{n}^{*}\right)\right\}_{n \in \mathbb{N}}$, where $e_{n}^{*}$ are the dual functionals in $l_{\infty}^{*}(\mathbb{N})$, is a countable boundary of $\left(X,\|\cdot\|_{1}\right)$.

(i) $\Rightarrow$ (iv) The separability of $X$ follows from a result of Gilles Godefroy [4]. Recall that in the course of our proof of (ii) $\Rightarrow$ (i) we showed that $X$ is isomorphic to some $Y \subset l_{\infty}(\mathbb{N})$ such that every $y \in Y$ attains its norm, i.e., $y(n)=\|y\|_{\infty}$ for some $n \in \mathbb{N}$. Consider a decreasing sequence $\delta_{n} \downarrow 0$ and an isomorphism $S: Y \rightarrow Z, Z \subset l_{\infty}(\mathbb{N})$ defined by

$$
S(y)(n)=\left(1+\delta_{n}\right) y(n) \text {. }
$$

We will renorm the space $\left(Z,\|\cdot\|_{\infty}\right)$ by the desired $C^{\infty}$-smooth norm. Note that for each $y \in Y$ with $\|y\|_{\infty}=1$ there is an $n \in \mathbb{N}$ satisfying $|y(n)|=$ 1. Therefore for each $z \in Z$ there exists $n_{z} \in \mathbb{N}$ satisfying $\left|S^{-1}(z)\left(n_{z}\right)\right|=$ $\left\|S^{-1}(z)\right\|_{\infty}$ and thus

$$
\left|z\left(n_{z}\right)\right|=\left(1+\delta_{n_{z}}\right)\left\|S^{-1}(z)\right\|_{\infty}
$$

and

$$
|z(k)| \leq\left(1+\delta_{n_{z}+1}\right)\left\|S^{-1}(z)\right\|_{\infty} \text { for } k>n_{z} .
$$

Take a sequence $\left\{b_{n}\right\}_{n \in \mathbb{N}}$ of $C^{\infty}$-smooth bump functions $b_{n}: \mathbb{R} \rightarrow \mathbb{R}, b_{n} \geq$ $0, \int_{-\infty}^{\infty} b_{n}(t) d t=1, \operatorname{supp}\left(b_{n}\right) \subset\left[\left(\delta_{n+1}-\delta_{n}\right) / 4,\left(\delta_{n}-\delta_{n+1}\right) / 4\right]$. Define a nondecreasing sequence $\left\{F_{n}\right\}_{n=0}^{\infty}$ of convex functions on $l_{\infty}(N)$ by the inductive formula

$$
\begin{gathered}
F_{0}=\|\cdot\|_{\infty}, \\
F_{n}(z)=\int_{\left(\delta_{n+1}-\delta_{n}\right) / 4}^{\left(\delta_{n}-\delta_{n+1}\right) / 4} F_{n-1}\left(z+t e_{n}\right) b_{n}(t) d t .
\end{gathered}
$$

Suppose $\left\|S^{-1}(z)\right\|_{\infty} \geq 1$ and $\rho=\left(\delta_{n_{z}}-\delta_{n_{z}+1}\right) / 4,\|y-z\|_{\infty}<\rho$. Then for $k>n_{z}$ we have

$$
\begin{aligned}
F_{k}(y)=\int_{\left(\delta_{k+1}-\delta_{k}\right) / 4}^{\left(\delta_{k}-\delta_{k+1}\right) / 4} \cdots \int_{\left(\delta_{n_{2}+1}-\delta_{n_{z}}\right) / 4}^{\left(\delta_{n_{z}}-\delta_{n_{z}+1}\right) / 4} \cdots \\
\cdot \int_{\left(\delta_{2}-\delta_{1}\right) / 4}^{\left(\delta_{1}-\delta_{2}\right) / 4}\left\|y+t_{1} e_{1}+\cdots+t_{k} e_{k}\right\|_{\infty} b_{1}\left(t_{1}\right) \cdots b_{k}\left(t_{k}\right) d t_{1} \cdots d t_{k} .
\end{aligned}
$$

Since $-\rho \leq t_{l} \leq \rho$ for $n_{z} \leq l \leq k$ and $y\left(n_{z}\right)-y(l) \geq 4 \rho$, we have that

$$
\left\|y+t_{1} e_{1}+\cdots+t_{k} e_{k}\right\|_{\infty}=\left\|y+t_{1} e_{1} \cdots+t_{n_{z}} e_{n_{z}}\right\|_{\infty} .
$$

Consequently

$$
\begin{aligned}
F_{k}(y)=F_{n_{z}}(y)=\int_{\left(\delta_{n_{z}+1}-\delta_{n_{z}}\right) / 4}^{\left(\delta_{n_{z}}-\delta_{n_{z}+1}\right) / 4} \ldots \\
\qquad \int_{\left(\delta_{2}-\delta_{1}\right) / 4}^{\left(\delta_{1}-\delta_{2}\right) / 4} \max \left\{\left|y(1)+t_{1}\right|,\left|y(2)+t_{2}\right|, \ldots,\left|y\left(n_{z}\right)+t_{n_{z}}\right|\right\} \\
\cdot b_{1}\left(t_{1}\right) \cdots b_{n_{z}}\left(t_{n_{z}}\right) d t_{1} \cdots d t_{n_{z}}
\end{aligned}
$$

for $\|z-y\|_{\infty}<\rho$ and $k \geq n_{z}$. It follows that the convex function $F$ on $Z$ defined by $F=\sup _{n \in \mathbb{N}}\left(F_{n}\right)$ is locally dependent on finitely many coordinates (namely the functionals $e_{1}^{*}, \ldots, e_{n_{z}}^{*}$ ) and $C^{\infty}$-smooth on $\left\{z \in \mathbb{Z} ;\|z\|_{\infty}>\right.$ 
$\left.1+\delta_{1}\right\}$. Notice that $F_{k}(z) \leq\|z\|_{\infty}+\frac{\delta_{1}-\delta_{2}}{4}$ for arbitrary $z \in l_{\infty}(N)$ and $k \in N$. Applying the Implicit Function Theorem, we obtain that the Minkowski functional of the set $\left\{z \in Z, F(z) \leq 1+2 \delta_{1}\right\}$ introduces a $C^{\infty}$ norm on $Z$ that locally depends on finitely many coordinates.

Corollary 2 (Fonf). Suppose that any of the equivalent statements (i)-(iv) is satisfied for a normed space $(X,\|\cdot\|)$. In particular, suppose that the set ext $B_{X}$ * is countable. Then $X$ contains an isomorphic copy of $c_{0}$.

Proof. The space satisfies (iii). By the result of Pechanec, Whitfield, and Zizler [11] we obtain the conclusion immediately.

Corollary 3 (Haydon). Every space $C(K)$ where $K$ is a countable compact set admits an equivalent $C^{\infty}$-smooth norm.

Proof. Immediate from Theorem 1, since the Dirac functionals form a countable boundary for the supremum norm on $C(K)$.

Corollary 4. Every normed space $(X,\|\cdot\|)$ with countable algebraic basis admits an equivalent $C^{\infty}$-smooth norm.

Proof. Let $\left\{x_{n}\right\}_{n \in \mathbb{N}}$ be an algebraic basis. Following Vanderwerff [14] and [9], we imbed $X$ into $C[0,1]$ and renorm this latter space by a norm $|\cdot|$ so that there exists a sequence of finite-dimensional projections $\left\{Q_{n}\right\}_{n \in \mathbb{N}},\left|Q_{n}\right| \leq 1$, $Q_{k} Q_{j}=Q_{j} Q_{k}=Q_{\min (k, j)}$ such that

$$
\left.Q_{k}\right|_{\operatorname{span}\left\{x_{1}, \ldots, x_{n}\right\}}=\left.\operatorname{Id}\right|_{\operatorname{span}\left\{x_{1}, \ldots, z_{n}\right\}}
$$

for $k$ larger than $k(n), n$ arbitrary. This allows us to cover the boundary of $(X,|\cdot|)$ by the sequence of compacts $\left\{K_{n}\right\}_{n \in \mathbb{N}}, K_{n}=Q_{n}^{*}\left(C[0,1]^{*}\right) \cap S_{X^{*}}$. By our theorem, we are done.

Recall that the Talagrand operator on $C_{0}(K)$ space, $K$ a compact set, is a continuous linear mapping $T: C_{0}(K) \rightarrow c_{0}(K)$ such that for every $f \in C_{0}(K)$ there exists some $k \in K$ so that

$$
|f(k)|=\|f\|_{\infty} \text { and }|f(k)+T f(k)|>|f(k)| .
$$

It follows easily that the norm $\||\cdot|\|$ defined on $C_{0}(K)$ by \|\|$f\|\|=\|f\|_{\infty}+$ $\|T f\|_{\infty}$ depends locally on finitely many coordinates. This fact allows us to drop the assumption of scatteredness of $K$ in the result of Haydon [6].

Corollary 5. Suppose the space $C_{0}(K)$, where $K$ is a locally compact set, admits a Talagrand operator into $c_{0}(K)$. Then $C_{0}(K)$ has an equivalent $C^{\infty}$-smooth norm.

Proof. Every separable subspace of $C_{0}(K)$ admits a norm depending locally on finitely many coordinates. By Theorem 1 , such a space can be renormed by an equivalent $C^{\infty}$-smooth norm, therefore it is an Asplund space. Hence the whole $C_{0}(K)$ is an Asplund space. Thus $K$ is a scattered locally compact set (see [1], Lemma 8.3, page 258) and we are ready to use Haydon's result [6] on such spaces, which guarantees the existence of $C^{\infty}$-smooth renorming.

\section{ACKNOWLEDGMENT}

The author would like to thank Dr. V. Zizler for his encouragement and valuable suggestions. 


\section{REFERENCES}

1. R. Deville, G. Godefroy, and V. Zizler, Smoothness and renormings in Banach spaces, Pitman Monographs Surveys Pure Appl. Math., vol. 64, Longman Sci. Tech., Harlow, 1993.

2. M. Fabian, J. H. M. Whitfield, and V. Zizler, Norms with locally Lipschitzian derivatives, Israel J. Math. 44 (1983), 262-276.

3. V. P. Fonf, One property of Lindenstrauss-Phelps spaces, Funktsional. Anal. i Prilozhen. 13 (1977), 79-80.

4. G. Godefroy, Some applications of Simon's inequality, preprint.

5. B. V. Godun, B. L. Lin, and S. L. Troyanski, On the strongly extreme points of convex bodies in separable Banach spaces, Proc. Amer. Math. Soc. 114 (1992), 673-675.

6. R. G. Haydon, Infinitely differentiable norms on certain Banach spaces, preprint.

7. __ Normes et partitions de l'unité indéfiniment différentiables sur certains espaces de Banach, C. R. Acad. Sci. Paris Ser. I 315 (1992), 1175-1178.

8.

9. W. B. Johnson, H. P. Rosenthal, and M. Zippin, On bases, finite dimensional decompositions and weaker structures in Banach spaces, Israel J. Math. 9 (1971), 488-506.

10. J. Lindenstrauss and R. R. Phelps, Extreme points properties of convex bodies in reflexive Banach spaces, Israel J. Math. 6 (1968), 39-48.

11. J. Pechanec, J. H. M. Whitfield, and V. Zizler, Norms locally dependent on finitely many coordinates, An. Acad. Brasil. Ciênc. 53 (1981), 415-417.

12. M. Talagrand, Renormages de quelques $C(K)$, Israel J. Math. 54 (1986), 323-334.

13. S. Troyanski, Gâteaux differentiable norms in $L_{p}$, Math. Ann. 287 (1990), 221-227.

14. J. Vanderwerff, Frechet differentiable norms on spaces of countable dimension, Arch. Math. $\mathbf{5 8}$ (1992), 471-476.

15. J. H. M. Whitfield and V. Zizler, Extremal structure of convex sets in spaces not containing $c_{0}$, Math. Z. 197 (1988), 219-221.

Department of Mathematics, University of Alberta, Edmonton, Canada T6G 2G1

E-mail address: phajek@vega.math.ualberta.ca 